\title{
Derivation of the Holographic Dual of a Planar Conformal Field Theory in 4D
}

\author{
Nikolay Gromov* \\ Mathematics Department, King's College London, The Strand, London WC2R 2LS, United Kingdom \\ and St. Petersburg INP, Gatchina 188 300, St. Petersburg, Russia \\ Amit Sever \\ School of Physics and Astronomy, Tel Aviv University, Ramat Aviv 69978, Israel \\ and CERN, Theoretical Physics Department, 1211 Geneva 23, Switzerland
}

(Received 24 April 2019; published 23 August 2019)

\begin{abstract}
We present the first-principles derivation of a weak-strong duality between the fishnet theory in four dimensions and a discretized stringlike model living in five dimensions. At strong coupling, the dual description becomes classical and we demonstrate explicitly the classical integrability of the model. We test our results by reproducing the strong coupling limit of the four-point correlator computed before nonperturbatively from the conformal partial wave expansion. Because of the extreme simplicity of our model, it could provide an ideal playground for holography with no supersymmetry. Furthermore, since the fishnet model and $\mathcal{N}=4$ super Yang-Mills theory are continuously linked, our consideration could shed light on the derivation of AdS/CFT for the latter. For simplicity, in this Letter we restrict our considerations to a large subset of all states.
\end{abstract}

DOI: 10.1103/PhysRevLett.123.081602

Introduction.-In recent years, the ideas of holography [1-3] conquered almost all corners of theoretical physics. The idea that some (or any?) strongly coupled quantum system with many degrees of freedom should have an alternative dual description in terms of the gravity or string theory in a higher dimensional spacetime is becoming more and more popular. Despite this enormous attention the holographic principle has received in the last two decades, we are still lacking the first-principles derivation of it. There are, however, numerous and extremely nontrivial tests of the duality. Because of its strong-weak character, it is very hard to produce these tests. In some special models, such as $\mathcal{N}=4$ super Yang-Mills (SYM) theory, tools such as supersymmetric localization, or integrability, provide ways to compute observables for arbitrary coupling strengths and compare with the holographic predictions.

In this Letter, we will provide the first-principles derivation of a holographic dual of the "fishnet model" [4]

$$
\mathcal{L}_{4 d}=N \operatorname{tr}\left[\left|\partial \phi_{1}\right|^{2}+\left|\partial \phi_{2}\right|^{2}+(4 \pi)^{2} \xi^{2} \phi_{1}^{\dagger} \phi_{2}^{\dagger} \phi_{1} \phi_{2}\right],
$$

in the planar expansion where $N$ is taken large, while $\xi^{2}$ is held fixed but arbitrary (Here, we have suppressed double

Published by the American Physical Society under the terms of the Creative Commons Attribution 4.0 International license. Further distribution of this work must maintain attribution to the author(s) and the published article's title, journal citation, and DOI. Funded by SCOAP . trace interactions that are not relevant nonperturbatively $[5,6]$.$) . Here, \phi_{1,2}$ are two $N \times N$ complex scalar fields. The model can be obtained from $\mathcal{N}=4$ SYM theory in a double scaling limit and was shown to be conformal and integrable in the planar limit $[4,5,7,8]$. This breaks the $\mathfrak{S}_{\mathfrak{t}} *(4 \mid 4)$ superconformal symmetry down to $\mathfrak{t}(1){ }_{1} \times$ $\mathfrak{t}(1)_{2} \times \mathfrak{s} \mathfrak{o}(1,5)$, leaving us with no supersymmetry at all. Yet, being a solvable interacting conformal field theory (CFT) in four dimensions, this model attracted a lot of attention. In particular, one can compute the spectrum of anomalous dimensions [7] as well as some structure constants and correlation functions [6] at any $\xi$. In all of these cases, there were indications of the existence of the holographic dual- the scaling dimensions $\Delta$ generically scale as $\xi$ and the four-point correlation functions behave as $e^{-\xi A(z, \bar{z})}$ [6]. At the same time, these indications were somehow puzzling, as the dual description of $\mathcal{N}=4$ SYM theory become weakly coupled at infinitely large $\lambda$, whereas the fishnet model obtained in the opposite $\sqrt{\lambda}=R_{\text {AdS }}^{2} / l_{s}^{2} \rightarrow 0$ limit [4]. Furthermore, the corresponding deformation is known to produce tachyonic instability in the string background [9]. As a result, it is not clear how to link the holographic string description of $\mathcal{N}=4$ SYM theory to that of the fishnets or even if that is possible at all.

Indeed, the dual description presented here is not in terms of a smooth string. Instead, we found a chain of $J$ particles or string bits with nearest-neighbor interactions. More precisely the dual model action functional $S_{\text {dual }}=\xi \int d t \sum_{i} L_{i}$, is given in terms of the Lagrangian density 


$$
L_{i}=-\frac{\dot{X}_{i}^{2}}{2}-\prod_{k=1}^{J}\left(-X_{k} X_{k+1}\right)^{-\frac{1}{J}}-\eta_{i}\left(X_{i}^{2}+R^{2}\right)+R^{2} .
$$

Here, $X_{i}(t) \in \mathbb{R}^{1,5}$ with $-++\cdots$ signature and $\eta_{i}(t)$, $R^{2}(t)$ are Lagrange multipliers. The world sheet coordinates $X(t)$ are further subjected to Virasoro-type constraints described below in (11) and (13). Note, in particular, that the square root of the 't Hooft coupling $\xi$ stands in front of the action and plays the role of $1 / \hbar$.

The field $R(t)$ looks like an anti-de Sitter (AdS) radius in string units. It satisfies a dynamical evolution equation and will be set to zero consistently. The $X_{i}$ coordinates are not projective and hence the action $S_{\text {dual }}$ describes a discretized string propagating on the five-dimensional light cone of $\mathbb{R}^{1,5}$, subject to the Virasoro constraints. The fifth dimension naturally emerges when making the symmetries manifest. It is encoded in a nontrivial way in all the original $J$ fourdimensional degrees of freedom and is related to an emergent local scale invariance.

Below we give the derivation of this result and also show how one can reproduce the classical limit of the anomalous dimensions and also the four-point functions.

Derivation of the dual action.-One of the main features of the fishnet theory is the simple structure of its Feynman diagrams. In this Letter, we consider the $\mathfrak{t}(1)$ sector of the model, where the $\mathfrak{t}(1)_{1}$ charge is $J$ and the $\mathfrak{t}(1)_{2}$ charge is set to zero. It consists of all operators of the type $\left[\partial^{m} \phi_{1}^{J}\left(\phi_{2} \phi_{2}^{\dagger}\right)^{n} \ldots\right]$, containing any number of derivatives, $J$-scalar fields $\phi_{1}$ and any neutral combination of $\phi_{2}$ and $\phi_{2}^{\dagger}$.

The Feynman diagrams that contribute to the correlation functions of these operators and their conjugates are of iterative fishnet type, after all $\phi_{2}$ 's annihilate with $\phi_{2}^{\dagger}$ (see Fig. 1). It is possible to resum, at least formally, infinitely

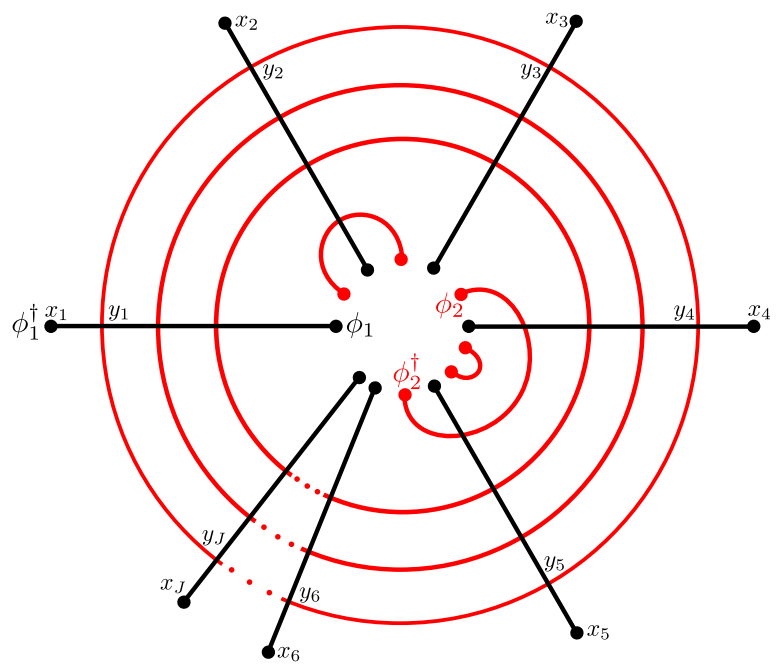

FIG. 1. Feynman diagram that contributes to the correlation function of an operator of the form $\operatorname{tr}\left[\partial^{m} \phi_{1}^{J}\left(\phi_{2} \phi_{2}^{\dagger}\right)^{n} \ldots\right]$ and $J \phi_{1}^{\dagger}$ scalars are all of fishnet type - made of an iterative wheel [10]. This structure can be resummed and leads to integrability [11]. many Feynman graphs by introducing the "graph-building" operator $\hat{B}$, defined by its integral kernel [4]

$$
B\left(\left\{\vec{y}_{i}\right\}_{i=1}^{J},\left\{\vec{x}_{j}\right\}_{j=1}^{J}\right)=\prod_{i=1}^{J} \frac{\xi^{2} / \pi^{2}}{\left(\vec{y}_{i}-\vec{y}_{i+1}\right)^{2}\left(\vec{x}_{i}-\vec{y}_{i}\right)^{2}} .
$$

Applying this operator once, we add one wheel to the graph in Fig. 1; thus the sum of all wheels inside the graph forms a geometric series

$$
\text { all wheels }=\frac{1}{1-\hat{B}} .
$$

We see that the zeros of the denominator play a special role. By diagonalizing $\hat{B}$, one finds that the eigenfunctions are parametrized by the continuous parameter $\Delta$, conjugated to the dilatation operator. The sum over the complete set of eigenfunctions will involve the integration over $\Delta$, which then can be computed by residues giving distinct meaning to those values of $\Delta$ where $\hat{B}=1$. Namely, those poles can be identified as the anomalous dimensions of the local operators. This procedure was exemplified in detail in [6]. The output of this discussion is that we need to solve $(\hat{B}-1) \Psi=0$, or, equivalently, acting on both sides with $\prod_{i} \square_{i}$, to cancel factors $1 /\left[4 \pi^{2}\left(x_{i}-y_{i}\right)^{2}\right]$, we find

$H \circ \Psi\left(\left\{x_{i}\right\}\right)=0, \quad H=\prod_{i=1}^{J} \vec{p}_{i}^{2}-\prod_{i=1}^{J} \frac{4 \xi^{2}}{\left(\vec{x}_{i}-\vec{x}_{i+1}\right)^{2}}$,

where $\vec{p}_{i} \equiv-i \vec{\partial}_{x_{i}}$. Under the operator-state correspondence, the wave function $\Psi$ is dual to a local operator. The key step in our derivation is to interpret (5) as the constraint appearing in a system with time reparametrization symmetry $t \rightarrow f(t)$, where $t$ is conjugate to $H$. To see this gauge symmetry manifestly, we write the Lagrangian corresponding to the Hamiltonian $H$ in (5). After solving for $\vec{p}_{i}$ in terms of $\dot{\vec{x}}_{i}=\left(\partial H / \partial \vec{p}_{i}\right)$, we arrive at

$$
L=\frac{2 J-1}{2^{\frac{2 J}{2 J-1}}}\left(\frac{1}{\gamma} \prod_{i=1}^{J} \overrightarrow{\dot{x}}_{i}^{2}\right)^{\frac{1}{2 J-1}}+\gamma \prod_{i=1}^{J} \frac{4 \xi^{2}}{\left(\vec{x}_{i}-\vec{x}_{i+1}\right)^{2}},
$$

where $\gamma$ transforms under reparametrization $t \rightarrow f(t)$ as $\gamma \rightarrow \gamma / f^{\prime}$ and (5) is the constraint that corresponds to fixing $\gamma=1$. Instead of fixing the gauge $\gamma=1$, it is more beneficial to eliminate the auxiliary field $\gamma$ by setting it to its extremum to obtain

$$
S=\xi \int L d t=2 J \xi \int\left(\prod_{i=1}^{J} \frac{\dot{\vec{x}}_{i}^{2}}{\left(\vec{x}_{i}-\vec{x}_{i+1}\right)^{2}}\right)^{\frac{1}{2 J}} d t,
$$

where the different branches of $\xi$ translate to the multivaluedness of the interaction term. One may draw analogies 
between (6) and (7) and the Polyakov and Nambu-Goto actions, respectively. In this analogy, the initial equation (5) corresponds to the Virasoro constraint. There is a number of significant observations one can make about (7). First, we see that the coupling $\xi$ plays the role of $1 / \hbar$ in the quasiclassical analysis, in accordance with the previous observations [6,7]. In particular, that explains the scaling $\Delta \sim \xi$ observed numerically in [7]. We note, however, that our starting point (3) contained $\xi^{2 J}$, implying that all roots $e^{\pi i n / J} \xi, n \in \mathbb{Z}$ should be considered. Different $n$ 's correspond to different branches in the spectrum, as we demonstrate in Sec. III. In addition to the time reparametrization symmetry, the action $S$ in the form (7) is also invariant under global conformal transformations, which is of course a highly expected property for a CFT dual. In the next section, we will make the conformal symmetry manifest by uplifting the action into a $6 \mathrm{D}$ embedding space.

Finally, we comment about an interpretation of the action (7). One may think of it as describing $J$ string bits $[12,13]$. Each bit corresponds to a spike of a holographic string that propagates at the AdS boundary, see Fig. 2. The dynamics of the string segment between the spikes results in the nearest-neighbors interaction of the model.

Embedding space formulation: As it is well known, the conformal group in 4D coincides with the group of rotations in $\mathbb{R}^{1,5}$. Under this identification, the flat space coordinate $x^{\mu=1, \ldots, 4}$ is mapped to the projective light cone of $\mathbb{R}^{1,5}$, parametrized by $X_{i}^{M}, M=-1,0,1, \ldots, 4$, as

$$
x_{i}^{\mu}=X_{i}^{\mu} / X_{i}^{+}, \quad X_{i}^{2}=0, \quad X_{i}^{+}=X_{i}^{0}+X_{i}^{-1} .
$$

After this change of variables, the action (7) becomes

$$
L=2 J\left(\prod_{i=1}^{J} \frac{\dot{X}_{i} \dot{X}_{i}}{-2 X_{i} X_{i+1}}\right)^{\frac{1}{2 J}}
$$

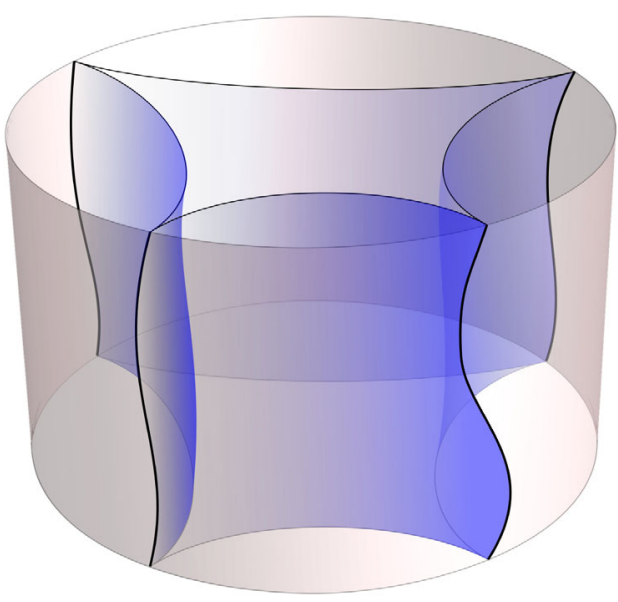

FIG. 2. Spiky string in AdS.
By uplifting to a projective space, we have introduced a new local gauge symmetry $X_{i} \rightarrow g_{i}(t) X_{i}$.

We now introduce auxiliary fields to disentangle the action as

$$
L=-\sum_{i}\left(\frac{\dot{X}_{i}^{2}}{2 \alpha_{i}}+\eta_{i} X_{i}^{2}+\gamma \prod_{k}\left(-X_{k} X_{k+1}\right)^{-\frac{1}{J}}\right),
$$

where the last term is independent of $i$. In order to get back to (9), one should extremize in $\alpha_{i}$ and $\gamma$, assuming $\prod \alpha_{i}=\gamma^{J}$. We also introduced the remaining constraint $X_{i}^{2}=0$ with the Lagrange multipliers $\eta_{i}$. The symmetries of the action are (1) manifest conformal symmetry, (2) time-dependent rescaling symmetry $X_{i} \rightarrow g_{i}(t) X_{i}, \alpha_{i} \rightarrow g_{i}^{2}(t) \alpha_{i}, \gamma \rightarrow \gamma \prod_{i} g_{i}^{2 / J}(t)$, and $\eta_{i} \rightarrow g_{i}^{-2}(t) \eta_{i}$, (3) time reparametrization symmetry $t \rightarrow f(t), \eta_{i} \rightarrow \eta_{i} / f^{\prime}$, and $\gamma \rightarrow \gamma / f^{\prime}$, and (4) translation along the chain $X_{i} \rightarrow X_{i+1}$. To fix the gauge symmetries, we can set $\alpha_{i}=\gamma=1$, leading to the constraints

$$
\dot{X}_{k}^{2}=2 \prod_{i}\left(-X_{i} X_{i+1}\right)^{-\frac{1}{J}} \equiv \mathcal{L}, k=1, \ldots, J,
$$

which is very reminiscent of the Virasoro constraints in the conformal gauge, telling us that the energy density is zero along the string. Finally, we notice that there is still one remaining gauge symmetry left $t \rightarrow f(t), X_{i} \rightarrow$ $X_{i} / \sqrt{f^{\prime}}, \eta_{i} \rightarrow \eta_{i} / f^{\prime}$, which we fix by further imposing $\sum \eta_{i}=J$, with the Lagrange multiplier $R^{2}$, leading to (2). This action together with (11) is our main result (alternative gauge choice $\mathcal{L}=1$ could be convenient too).

Equations of motion: The variation of (2) with respect to $X_{i}$ gives

$$
\ddot{X}_{i}=2 \eta_{i} X_{i}-\frac{\mathcal{L}}{2}\left(\frac{X_{i+1}}{X_{i+1} X_{i}}+\frac{X_{i-1}}{X_{i} X_{i-1}}\right) .
$$

By contracting (12) with $X_{i}$ and using that $X_{i}^{2}=0$, we arrive back at (11). Contracting (12) with $\dot{X}_{i}$, however, leads to the secondary constraint that is analogous of the second Virasoro constraint, imposing that

$$
\frac{\dot{X}_{i} X_{i+1}}{X_{i} X_{i+1}}+\frac{\dot{X}_{i} X_{i-1}}{X_{i} X_{i-1}}=-\partial_{t} \log \mathcal{L}
$$

does not depend on the site index $i$. (The relative sign may look strange. However, in the continuum limit, the analog of the rhs has the effect of correcting it [14].)

Finally, $\eta_{i}$ can be extracted from the derivative of (13). Instead, we eliminate $\eta_{i}$ by introducing the $\mathrm{SO}(1,5)$ charge density $q_{i}^{M N}=2 \dot{X}_{i}^{[M} X_{i}^{N]}$. The equation of motion (12) can be equivalently written as 


$$
\dot{q}_{i}=\frac{\mathcal{L}}{2}\left(j_{i+1}-j_{i}\right), \quad j_{i}^{M N}=2 \frac{X_{i-1}^{[M} X_{i}^{N]}}{X_{i-1} X_{i}},
$$

where $j_{i}$ can be interpreted a $\operatorname{SO}(1,5)$ current density. The $\mathrm{SO}(1,5)$ charge is given by $Q^{M N} \equiv \xi \mathcal{Q}^{M N}=\xi \sum_{i} q_{i}^{M N}$. We can always assume that $\mathcal{Q}^{M N}$ is block diagonal, with nonzero elements $\mathcal{Q}^{-1,0}=i \mathcal{D}, \mathcal{Q}^{1,2}=\mathcal{S}_{1}$, and $\mathcal{Q}^{3,4}=\mathcal{S}_{2}$, where $\mathcal{D}=\Delta / \xi$ and $\mathcal{S}_{a}=S_{a} / \xi$ are the appropriate notations for the large $\xi$ classical limit.

After introducing $R^{2}$, we are no longer constrained to the light cone and one could be worried about the consistency of the initial condition $X_{i}^{2}=-R^{2}=0$. By contracting (12) with $X_{i}$, we get $2 \partial_{t}^{2} R^{2}=\eta_{i} R^{2}$. Since $\sum_{i} \eta_{i}=J$ we obtain $2 \partial_{t}^{2} R^{2}=R^{2}$, meaning that once we set $R=0$ at some moment of time it will stay so forever.

Integrability: Our fish chain model at $R=0$ is dual to the integrable fishnet model and hence it is expected to be integrable too. Similar to the Toda chain, we find a pair of space- and timelike connections, dependant on the spectral parameter $u, \mathbb{L}_{i}(u)$ and $\mathbb{V}_{i}(u)$, that satisfy the zero curvature condition [15]

$$
\dot{\mathbb{L}}_{i}=\mathbb{V}_{i+1} \mathbb{\mathbb { L }}_{i}-\mathbb{L}_{i} \mathbb{V}_{i}
$$

This condition ensures that each coefficient of the polynomial $\mathbb{T}(u)=\operatorname{tr} \Omega(u)$, where $\Omega \equiv \mathbb{L}_{J}, \ldots, \mathbb{L}_{2} \mathbb{L}_{1}$, gives an integral of motion, constant in time on equations of motions. In the irreducible representation (irrep) 6 of $\mathrm{SO}(1,5)$, these matrices are

$$
\mathbb{L}_{i}^{\mathbf{6}}=u^{2}+u q_{i}+\frac{q_{i}^{2}}{2}, \quad \mathbb{V}_{i}^{\mathbf{6}}=\frac{j_{i}}{u} \frac{\mathcal{L}}{2} .
$$

To derive the discrete zero curvature condition (15), we use (14) and the identity $\left(q_{i}^{2}\right)^{M N}=-\mathcal{L} X_{i}^{M} X_{i}^{N}$, which implies, using (12), that $\partial_{t} q_{i}^{2}=\mathcal{L}\left(j_{i+1} q_{i}-q_{i} j_{i}\right)$ and $j_{i+1} q_{i}^{2}-q_{i}^{2} j_{i}=0$. Interestingly, the constraint (11) results in the relation $\mathbb{T}^{6}(0)=1$. Similar to (16), the space- and timelike connections in the irrep 4 take the form $\mathbb{L}_{k}^{4}=u-$ $(i / 2) q_{k}^{M N} \Sigma_{M N}$ and $\mathbb{V}_{k}^{4}=-(i \mathcal{L} / 4 u) j_{k}^{M N} \Sigma_{M N}$, where $\Sigma_{M N}$ are the $6 \mathrm{D} \sigma$ matrices. $\mathbb{L}^{\mathbf{6}}$ can be constructed from $\mathbb{L}_{k}^{4}$ by projecting $\mathbb{L}_{k}^{4} \otimes \mathbb{L}_{k}^{4}$ on the $\mathbf{6}$. Finally, one can show [14] that the Poisson bracket $\{\mathbb{T}(u), \mathbb{T}(v)\}=0$.

The key objects in integrability are the four quasimomenta $p_{a}$, which are defined as $\operatorname{det}\left[\Omega^{\mathbf{4}}(u)-u^{J} e^{i p_{a}(u)}\right]=0$. Their large $u$ asymptotic is determined by the global charges $p_{a} \simeq\left[\left( \pm \Delta \pm S_{1} \pm S_{2}\right) / 2 \xi u\right]$. At the origin $p_{a}$ 's have a logarithmic singularity $\pm i J \log u$. In addition, $p_{a}(u)$ has square-root singularities, coming from the diagonalization procedure. Together they form an algebraic curve, whose genus depends on the number of degrees of freedom. We expect the number of cuts to be equal to the number of independent cross ratios for $2 J$ points (i.e., 2 for $J=2$ and $8 J-15$ for $J>2$ ). Each $a$ cycle on the curve corresponds to an action variable $I_{a} \equiv(\xi / \pi i) \oint_{a} p(u) d u$. The action variables are expected to become integers in the BohrSommerfeld quantization procedure. We postpone more detailed investigation of the algebraic curve and separation of variables in this model for the future [14].

Explicit example.-First we consider the simplest case where $J=2$. This case was studied in detail at the quantum level, in particular, the spectrum is know exactly as [8]

$\Delta_{t=2 / 4}=2+\sqrt{\left(S_{1}+1\right)^{2}+1 \mp 2 \sqrt{\left(S_{1}+1\right)^{2}+4 \xi^{4}}}$,

where \pm corresponds to the twist $t=2$ and twist $t=4$ branches in the spectrum. The four-point function was computed in [6] as an infinite sum. In the classical limit, it was shown to sit on saddle points with classical dimension and spins that are related to the two conformal cross ratios as

$$
S_{\mathrm{cl}}^{2}= \pm \frac{4 \xi^{2} \theta^{2}}{\theta^{2}+\rho^{2}} \quad \text { and } \quad \Delta_{\mathrm{cl}}^{2}=S_{\mathrm{cl}}^{2} \mp 4 \xi^{2},
$$

where the second relation follows from (17). Here, $\rho$ and $\theta$ parametrize the two conformal cross ratios $u=[4 /(\cos \theta-$ $\left.\cosh \rho)^{2}\right]$ and $v=\left\{\left[(\cos \theta+\cosh \rho)^{2}\right] /\left[(\cos \theta-\cosh \rho)^{2}\right]\right\}$. Furthermore, the four-point correlation function itself takes the form $e^{-\xi A_{\mathrm{cl}}}\left(e^{i \xi A_{\mathrm{cl}}}\right)$ for $t=2(t=4)$, with $A_{\mathrm{cl}}=2 i \sqrt{\theta^{2}+\rho^{2}}$. Next, we try to reproduce these data from our classical dual description.

For $J=2$ we have two $6 \mathrm{D}$ null vectors, $X_{1}(t)$ and $X_{2}(t)$. Using global symmetries we can always go to the center of mass frame and set the last two components to zero $X_{1,2}=(r / \sqrt{2})(\cosh s, \sinh s, \pm \cos \phi$, $\mp \sin \phi, 0,0)$. In this parametrization, the coordinates $s$ and $\phi$ are conjugate to conserved charges, $\mathcal{D}=i r^{2} \dot{s}$ and $\mathcal{S}_{1}=r^{2} \dot{\phi}$. The constraint (11) gives $\pm 4=r^{4}\left(\dot{s}^{2}+\dot{\phi}^{2}\right)=\mathcal{D}^{2}+\mathcal{S}_{1}^{2}$, where the \pm sign comes from the different choice of the branch of the root on the rhs of (11). It perfectly reproduces the spectrum [(17) and (18)] in the classical limit where $S_{1}, \Delta \sim \xi \rightarrow \infty$, with different twists $t=2,4$ corresponding to different branches of the interaction term. Like in $\mathcal{N}=4$ SYM theory, the strong coupling limit of a correlator is expected to be given by $e^{i S}$ on a classical solution in the dual model. The classical action, with the constraint taken into account, becomes $S=\mp 4 \xi \int\left(d t / r^{2}\right)$. We see that it is beneficial to define the proper time $d \tau=\left[d t / r^{2}(t)\right]$, in terms of which

$$
S=\mp 4 \xi \tau, \quad s=-i \mathcal{D} \tau, \quad \phi=\mathcal{S}_{1} \tau .
$$

Next, computing the cross ratios between $X_{1,2}(\tau=0)$ and $X_{1,2}(\tau=T)$, we find in our parametrization $\theta=\mathcal{S}_{1} T$ and $\rho=i \mathcal{D} T$. Next, solving for $\mathcal{S}_{1}$ and $T$, with the constraint $\mathcal{S}_{1}^{2}-\mathcal{D}^{2}= \pm 4$, we find $T^{2}= \pm \frac{1}{4}\left(\theta^{2}+\rho^{2}\right)$ and $\mathcal{S}_{1}^{2}=$ $\pm\left[4 \theta^{2} /\left(\theta^{2}+\rho^{2}\right)\right]$, leading via (19) to $e^{i S}=e^{-2 i \xi \sqrt{\theta^{2}+\rho^{2}}}$ 


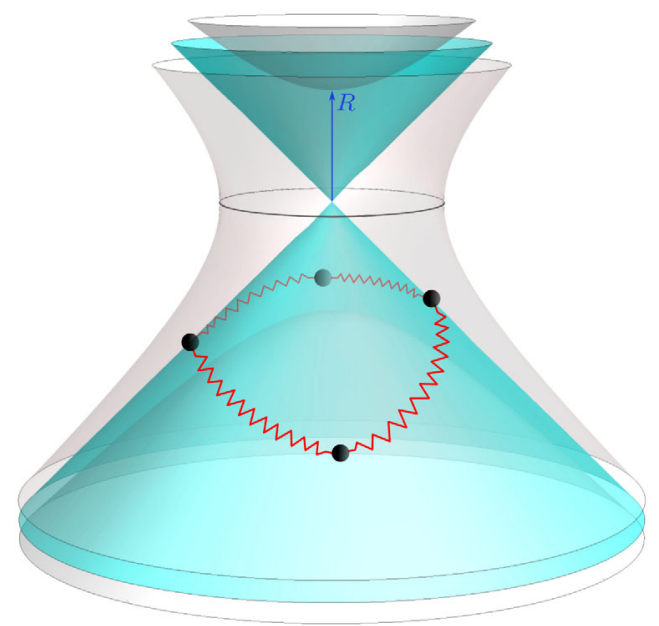

FIG. 3. Chain of $J$ particles on the light cone of $\mathbb{R}^{1,5}$.

and $e^{i S}=e^{-2 \xi \sqrt{\theta^{2}+\rho^{2}}}$ for $t=2$ and $t=4$ correspondingly, in perfect agreement with [6].

Discussion and speculation.-There are two fundamentally important properties of the fish chain model (2). First, the square root of the ' $t$ Hooft coupling constant $\xi$ stands in front of the action, playing the role of $1 / \hbar$. It emerged naturally from our interpretation of the graph-building operator. Second, the fish chain propagates in five-dimensional target space, see Fig. 3. The fifth dimension has emerged from the principle of realizing all symmetries in a manifestly covariant way. It may be thought of as a concrete realization of the holographic map and the original prediction of 't Hooft [16].

Going away from the model (1), one may add back the rest of the fields of $\mathcal{N}=4 \mathrm{SYM}$ theory in a controlled expansion around the fishnet limit [17] and incorporate their effect on the dual fish chain. Such expansion may open the path for a rigorous proof of AdS/CFT. One way in which this path may materialize is the following. The radius $R^{2}$ came about as a Lagrange multiplier, associated with a global rescaling gauge symmetry. It is consistently set to zero in the relevant classical solutions discussed above. If the correction away from the fishnet limit will stabilize it at some fixed $R^{2}>0$, then the discretized string will propagate in $\mathrm{AdS}_{5}$ instead of the light cone of $\mathbb{R}^{1,5}$. Moreover, for fixed $R^{2}>0$, the model has a smooth large $J$ continuum limit where $X_{i}\left(X_{i+1}-X_{i}\right) \simeq \frac{1}{2} \epsilon^{2} X^{\prime \prime}$. As a result, a new local time reparametrization symmetry emerges. Using similar manipulations to the ones in Sec. II, one arrives at a string action in $\mathrm{AdS}_{5}$ that is subject to the standard local Virasoro constraint, including a constant contribution from the extension of the string on the sphere [14]. In the $J \rightarrow \infty$ limit we may also be able to find a connection with the proposal of [18].

There are many future directions to pursue, some of them we list as follows. (i) One would like to extend the fish chain model away from the $\mathfrak{t}(1)$ sector by incorporating extra $\phi_{2}$ fields. (ii) Similar to the string in $\operatorname{AdS}_{5} \times S^{5}$, our action is highly nonlinear and its quantization is not notably straightforward. To start, one may incorporate quasiclassical corrections. (iii) Systematic $1 / N$ expansion should lead to fish chain interaction vertices. (iv) The open fish chain version of the model, dual to Wilson lines in the ladder limit [19], can be obtained from the derivation above by adding two more sites, replacing $\vec{p}_{1, J+2}^{2} \rightarrow \vec{p}_{1, J+2}^{2}+m^{2}$ and taking the large mass limit. (v) We expect the fish chain to exhibit $T$ duality. (vi) The simplicity of the classical model could help with the separation of variables approach $[20,21]$ to the correlation functions in $\mathcal{N}=4$ SYM theory.

Finally, analogous fishnet diagrams also exist in two, three, and six dimensions [11], and one may try to derive their duals. In particular, one may consider the large twist limit of the Aharony-Bergman-Jafferis-Maldacena model $[22,23]$ or more general fishnets, which could also include fermions [24].

We thank D. Anninos, B. Basso, A. Cavaglià, G. Korchemsky, F. Levkovich-Maslyuk, I. Kostov, S. Lukyanov, F. Smirnov, K. Zarembo, B. Vicedo, S. Zhiboedov, and especially V. Kazakov for invaluable discussions. N. G. acknowledges the hospitality of the theory group at CERN during the main phase of this work. N. G. was supported by the STFC Grant (No. ST/P000258/1). A. S. was supported by the I-CORE Program of the Planning and Budgeting Committee, The Israel Science Foundation (1937/12).

*nikolay.gromov@kcl.ac.uk

†amit.sever@CERN.ch

[1] J. M. Maldacena, The Large N limit of superconformal field theories and supergravity, Int. J. Theor. Phys. 38, 1113 (1999); Adv. Theor. Math. Phys. 2, 231 (1998).

[2] S. S. Gubser, I. R. Klebanov, and A. M. Polyakov, Gauge theory correlators from noncritical string theory, Phys. Lett. B 428, 105 (1998).

[3] E. Witten, Anti-de Sitter space and holography, Adv. Theor. Math. Phys. 2, 253 (1998).

[4] Ö. Gürdogan and V. Kazakov, New Integrable 4D Quantum Field Theories from Strongly Deformed Planar $\mathcal{N}=4$ Supersymmetric Yang-Mills Theory, Phys. Rev. Lett. 117, 201602 (2016).

[5] J. Fokken, C. Sieg, and M. Wilhelm, Non-conformality of $\gamma_{i}$-deformed $N=4$ SYM theory, J. Phys. A 47, 455401 (2014).

[6] N. Gromov, V. Kazakov, and G. Korchemsky, Exact correlation functions in conformal fishnet theory, arXiv: 1808.02688 .

[7] N. Gromov, V. Kazakov, G. Korchemsky, S. Negro, and G. Sizov, Integrability of conformal fishnet theory, J. High Energy Phys. 01 (2018) 095.

[8] D. Grabner, N. Gromov, V. Kazakov, and G. Korchemsky, Strongly Gamma-Deformed $N=4$ SYM as an Integrable CFT, Phys. Rev. Lett. 120, 111601 (2018). 
[9] E. Pomoni and L. Rastelli, Large N field theory and AdS tachyons, J. High Energy Phys. 04 (2009) 020.

[10] H. B. Nielsen and P. Olesen, A Parton view on dual amplitudes, Phys. Lett. 32B, 203 (1970).

[11] A. B. Zamolodchikov, Fishnet diagrams as a completely integrable system, Phys. Lett. 97B, 63 (1980).

[12] O. Bergman and C. B. Thorn, String bit models for superstring, Phys. Rev. D 52, 5980 (1995).

[13] N. Dorey, A spin chain from string theory, arXiv:0805.4387.

[14] N. Gromov and A. Sever, Quantum fishchain in $\mathrm{AdS}_{5}$, arXiv:1907.01001.

[15] L. Faddeev and L. Takhtajan, Hamiltonian Methods in the Theory of Solitons (Springer, Berlin, 1987), p. 592.

[16] G. 't Hooft, A planar diagram theory for strong interactions, Nucl. Phys. B72, 461 (1974).

[17] D. Bykov and K. Zarembo, Ladders for Wilson loops beyond leading order, J. High Energy Phys. 09 (2012) 057.

[18] B. Basso and D. 1. Zhong, Continuum limit of fishnet graphs and AdS sigma model, J. High Energy Phys. 01 (2019) 002.
[19] D. Correa, J. Henn, J. Maldacena, and A. Sever, The cusp anomalous dimension at three loops and beyond, J. High Energy Phys. 05 (2012) 098.

[20] A. Cavaglià, N. Gromov, and F. Levkovich-Maslyuk, Quantum spectral curve and structure constants in $\mathcal{N}=4$ SYM: Cusps in the ladder limit, J. High Energy Phys. 10 (2018) 060.

[21] A. Cavaglià, N. Gromov, F. Levkovich-Maslyuk, and A. Sever (to be published).

[22] O. Aharony, O. Bergman, D. L. Jafferis, and J. Maldacena, $N=6$ superconformal Chern-Simons-matter theories, M2-branes and their gravity duals, J. High Energy Phys. 10 (2008) 091.

[23] J. Caetano, O. Grdoan, and V. Kazakov, Chiral limit of $\mathcal{N}=4$ SYM and ABJM and integrable Feynman graphs, J. High Energy Phys. 03 (2018) 077.

[24] V. Kazakov, E. Olivucci, and M. Preti, Generalized fishnets and exact four-point correlators in chiral $\mathrm{CFT}_{4}$, J. High Energy Phys. 06 (2019) 078. 